\title{
Progenitor Cell Engraftment
}

National Cancer Institute

\section{Source}

National Cancer Institute. Progenitor Cell Engraftment. NCI Thesaurus. Code C16034.

Establishment of a self replicating population of stem cells with subsequent repopulation of differentiated cell types. 